Analele Universităţii de Vest, Timişoara

Seria Matematică - Informatică LIV, 1, (2016), 17- 33

\title{
Extended Local Analysis of Inexact Gauss-Newton-like Method for Least Square Problems using Restricted Convergence Domains
}

Ioannis K. Argyros and Santhosh George

\begin{abstract}
We present a local convergence analysis of inexact Gauss-Newton-like method (IGNLM) for solving nonlinear leastsquares problems in a Euclidean space setting. The convergence analysis is based on our new idea of restricted convergence domains. Using this idea, we obtain a more precise information on the location of the iterates than in earlier studies leading to smaller majorizing functions. This way, our approach has the following advantages and under the same computational cost as in earlier studies: A large radius of convergence and more precise estimates on the distances involved to obtain a desired error tolerance. That is, we have a larger choice of initial points and fewer iterations are also needed to achieve the error tolerance. Special cases and numerical examples are also presented to show these advantages.
\end{abstract}

AMS Subject Classification (2000). 65H10, 65H05, 65J15, 65G99.

Keywords. Inexact Gauss-Newton-like method, Euclidean space, Nonlinear least squares problems, weak Lipschitz condition, weak and center-weak Lipschitz condition, local convergence, restricted convergence domains. 


\section{Introduction}

In this paper we are concerned with the problem of approximating a solution $x^{*}$ of the nonlinear least squares problem:

$$
\min G(x):=\frac{1}{2} F(x)^{T} F(x),
$$

where $F$ is Fréchet-differentiable defined on $\mathbb{R}^{n}$ with values in $\mathbb{R}^{m}, m \geq n$. The solution of these problems are very important in computational sciences and other disciplines $[2,9,10,12,15]$ and the solution of these problems can be found in closed form only in special cases. In computational sciences the practice of numerical analysis is essentially connected to variants of Newton's method [1]- [15]. In the present paper we consider the local convergence of inexact Gauss-Newton method (IGNM) for solving problem (1.1). Define the method as:

For $k=0$ step 1 until convergence do.

Find the step $\Delta_{k}$ which satisfies

$$
B_{k} \Delta_{k}=-F^{\prime}\left(x_{k}\right)^{T} F\left(x_{k}\right)+r_{k} \text {, where } \frac{\left\|r_{k}\right\|}{\left\|F^{\prime}\left(x_{k}\right)^{T} F\left(x_{k}\right)\right\|} \leq \eta_{k} .
$$

Set $x_{k+1}=x_{k}+\Delta_{k}$.

Here, $\left\{\eta_{k}\right\}$ is a sequence of forcing terms such that $\eta_{k} \in[0,1]$ for each $k$.

If $B_{k}=F^{\prime}\left(x_{k}\right)^{T} F\left(x_{k}\right)$, the process is called inexact Gauss-Newton method IGNM. Moreover, it is called inexact Gauss-Newton-like method IGNLM, if $B_{k}=B\left(x_{k}\right)$ approximates $F^{\prime}\left(x_{k}\right)^{T} F\left(x_{k}\right)$. There is a plethora of convergence results for IGNM and IGNLM under various Lipschitz-type conditions [1][15]. Recently, studies have been focused on the analysis of stopping residual controls $\frac{\left\|r_{k}\right\|}{\left\|F\left(x_{k}\right)\right\|} \leq \eta_{k}$ and its effect on convergence properties [1,4,6-9, 11, 12] by considering iterative form where a scaled residual control is performed at each iteration as follows:

For $k=0$ step 1 until convergence do.

Find the step $\Delta_{k}$ which satisfies

$$
B_{k} \Delta_{k}=-F\left(x_{k}\right)+r_{k}, \text { where } \frac{\left\|P_{k} r_{k}\right\|}{\left\|P_{k} F\left(x_{k}\right)\right\|} \leq \theta_{k} .
$$

Set $x_{k+1}=x_{k}+\Delta_{k}$. 
Here, $P_{k} \in L\left(\mathbb{R}^{m}, \mathbb{R}^{n}\right)$ for each $k$.

In this work, we are motivated by the works by J. Chen [6-8] and optimization considerations. We show the advantages as stated in the abstract of this paper by considering more precise convergence domains where the iterates are located than in earlier studies such as [6-8] leading to tighter majorizing sequences.

The paper is organized as follows. Some auxiliary results on MoorePenrose inverses and some Lemmas that shorten the proofs to follow are given in Section 2. The local convergence of IGNM and IGNLM is presented in Section 3. Special cases, numerical examples and favorable comparisons of our work over earlier ones such as $[6-8,11]$ are given in Section 4. Finally, the paper ends with a conclusion in Section 5.

\section{Auxiliary Results}

Let $U(x, r)$ and $\bar{U}(x, r)$ stand, respectively, for the open and closed ball in $\mathbb{R}^{n}$ with center $x$ and radius $r>0$. Let $\mathbb{R}^{m \times n}$ denote the set of all $m \times n$ matrices $A$. We denote by $A^{\dagger}$ the Moore-Penrose inverse of matrix $A[13,14]$. If $A$ has full rank, i.e., if $\operatorname{rank}(A)=\min \{m, n\}=n$, then $A^{\dagger}=\left(A^{T} A\right)^{-1} A^{T}$. Next, we present some standard Lemmas.

Assumption 2.1. [13]. Suppose that $A, E \in \mathbb{R}^{m \times n}, B=A+E$, $\left\|A^{\dagger}\right\|\|E\|<1$, $\operatorname{rank}(A)=\operatorname{rank}(B)$, then

$$
\left\|B^{\dagger}\right\| \leq \frac{\left\|A^{\dagger}\right\|}{1-\left\|A^{\dagger}\right\|\|E\|}
$$

Moreover, if $\operatorname{rank}(A)=\operatorname{rank}(B)=\min \{m, n\}$, then

$$
\left\|B^{\dagger}-A^{\dagger}\right\| \leq \frac{\sqrt{2}\left\|A^{\dagger}\right\|^{2}\|E\|}{1-\left\|A^{\dagger}\right\|\|E\|}
$$

Assumption 2.2. [13]. Suppose that $A, E \in \mathbb{R}^{m \times n}, B=A+E,\left\|E A^{\dagger}\right\|\|E\|<$ $1, \operatorname{rank}(A)=n$, then $\operatorname{rank}(B)=n$.

From now on $L_{0}, L$ and $L_{1}$ are positive integrable and nondecreasing functions on the interval $[0, r], r \in[0, R]$ for some fixed $R>0$. Next, we need results involving weak Lipschitz condition in the case $F^{\prime}(x)^{\dagger}=F^{\prime}(x)^{-1}$. Assumption 2.3. Suppose that: $F$ is continuously Fréchet-differentiable in $U\left(x^{\star}, r\right), F\left(x^{\star}\right)=0, F^{\prime}\left(x^{\star}\right)$ has full rank: 
(i) $F^{\prime}$ satisfies the center-Lipschitz condition with $\mathcal{L}_{0}$ average:

$\left\|F^{\prime}\left(x^{\theta}\right)-F^{\prime}\left(x^{\star}\right)\right\| \leq \int_{0}^{\theta s(x)} \mathcal{L}_{0}(u) d u$ for each $x \in U\left(x^{\star}, r\right), 0 \leq \theta \leq 1$,

where $x^{\theta}=x^{\star}+\theta\left(x-x^{\star}\right), s(x)=\left\|x-x^{\star}\right\|$ and $\mathcal{L}_{0}$ is a positive integrable function. Moreover, suppose that there exists $r_{0} \in(0, r)$ such that for $\beta=\left\|F^{\prime}\left(x^{\star}\right)^{\dagger}\right\|$,

$$
\beta \int_{0}^{r_{0}} \mathcal{L}_{0}(u) d u<1
$$

Then,

$$
\int_{0}^{1}\left\|F^{\prime}\left(x^{\theta}\right)-F^{\prime}\left(x^{\star}\right)\right\| s(x) d \theta \leq \int_{0}^{s(x)} \mathcal{L}_{0}(u)(s(x)-u) d u .
$$

and

$$
\left\|F^{\prime}(y)^{\dagger} F(x)\right\| \leq \frac{\left\|x-x^{\star}\right\|+\beta \int_{0}^{s(x)} \mathcal{L}_{0}(u)(s(x)-u) d u}{1-\beta \int_{0}^{s(y)} \mathcal{L}_{0}(u) d u}
$$

for each $x$ and $y \in U\left(x^{\star}, r_{0}\right)$.

(ii) If $F^{\prime}$ satisfies the radius Lipschitz condition with the $\mathcal{L}$ average:

$\left\|F^{\prime}(x)-F^{\prime}\left(x^{\theta}\right)\right\| \leq \int_{\theta s(x)}^{s(x)} \mathcal{L}(u) d u$ for each $x$ and $y \in U\left(x^{\star}, r_{0}\right), \quad 0 \leq \theta \leq 1$,

where $\mathcal{L}$ is a positive integrable function. Then,

$$
\int_{0}^{1}\left\|F^{\prime}(x)-F^{\prime}\left(x^{\theta}\right)\right\| s(x) d \theta \leq \int_{0}^{s(x)} \mathcal{L}(u) u d u .
$$

Moreover, suppose that

$$
L_{0}(u) \leq L(u)
$$

and (2.4) hold for each $u \in\left[0, r_{0}\right]$. Then,

$$
\begin{aligned}
\left\|F^{\prime}(x)^{\dagger} F(x)\right\| & \leq s(x)+\frac{\beta \int_{0}^{s(x)} \mathcal{L}(u) u d u-\beta \int_{0}^{s(x)}\left(\mathcal{L}(u)-\mathcal{L}_{0}(u) s(x) d u\right.}{1-\beta \int_{0}^{s(x)} \mathcal{L}_{0}(u) d u} \\
& \leq s(x)+\frac{\beta \int_{0}^{s(x)} \mathcal{L}(u) u d u}{1-\beta \int_{0}^{s(x)} \mathcal{L}_{0}(u) d u}
\end{aligned}
$$

for each $x \in U\left(x^{\star}, r_{0}\right)$. 
Proof. (i) See [6], but use (2.3) instead of (2.7) for inverses.

(ii) Using (2.7), we obtain in turn

$$
\begin{aligned}
\int_{0}^{1}\left\|F^{\prime}(x)-F^{\prime}\left(x^{\theta}\right)\right\| s(x) d \theta & \leq \int_{0}^{1} \int_{\theta s(x)}^{s(x)} \mathcal{L}(u) d u s(x) d \theta \\
& =\int_{0}^{s(x)} \mathcal{L}(u) u d u,
\end{aligned}
$$

which shows (2.8). Moreover, since $F^{\prime}\left(x^{\star}\right)$ has full rank, it follows from Lemmas 2.1, 2.2, 2.3 and

$$
\left\|F^{\prime}\left(x^{\star}\right)^{\dagger}\right\|\left\|F^{\prime}(x)-F^{\prime}\left(x^{\star}\right)\right\| \leq \beta \int_{0}^{s(x)} \mathcal{L}_{0}(u) d u<1 \text { for each } x \in U\left(x^{\star}, r_{0}\right)
$$

that $F^{\prime}(x)$ has full rank and

$$
\left\|\left[F^{\prime}(x)^{T} F^{\prime}(x)\right]^{-1} F^{\prime}(x)^{T}\right\| \leq \frac{\beta}{1-\beta \int_{0}^{s(x)} \mathcal{L}_{0}(u) d u} \text { for each } x \in U\left(x^{\star}, r_{0}\right) .
$$

Notice that we have the estimates

$$
F^{\prime}(y)^{\dagger} F^{\prime}\left(x^{\theta}\right)=I-F^{\prime}(y)^{\dagger}\left(F^{\prime}(y)-F^{\prime}\left(x^{\theta}\right)\right)
$$

and

$$
\begin{aligned}
F^{\prime}(y)^{\dagger} F(x) & =F^{\prime}(y)^{\dagger}\left(F(x)-F\left(x^{\star}\right)\right) \\
& =\int_{0}^{1} F^{\prime}(y)^{\dagger} F^{\prime}\left(x^{\theta}\right) d \theta\left(x-x^{\star}\right) .
\end{aligned}
$$

Then, in view of $(2.8),(2.11)-(2.13)$, we get in turn that

$$
\begin{aligned}
\left\|F^{\prime}(x)^{\dagger} F(x)\right\| & =\left\|\int_{0}^{1}\left(I-F^{\prime}(x)^{\dagger}\left(F^{\prime}(x)-F^{\prime}\left(x^{\theta}\right)\right)\right) d \theta\left(x-x^{\star}\right)\right\| \\
& \leq\left(1+\left\|F^{\prime}(x)^{\dagger}\right\| \int_{0}^{1}\left\|F^{\prime}(x)-F^{\prime}\left(x^{\theta}\right)\right\| d \theta\right)\left\|x-x^{\star}\right\|
\end{aligned}
$$

which implies (2.10).

$R E M A R K 2.4$. If $F^{\prime}$ satisfies the radius condition with $\mathcal{L}_{1}$ average on $U\left(x_{0}, r\right)$, then, $F^{\prime}$ satisfies the center Lipschitz condition with $\mathcal{L}_{0}$ average but not necessarily vice versa even if $\mathcal{L}_{0}=\mathcal{L}_{1}$. Notice also that in general

$$
\mathcal{L}_{0}(u) \leq \mathcal{L}_{1}(u) \text { and } L(u) \leq L_{1}(u) \text { for each } u \in\left[0, r_{0}\right]
$$


holds and $\frac{\mathcal{L}_{1}}{\mathcal{L}_{0}}$ can be arbitrarily large (see, the numerical examples at the end of the paper). If $\mathcal{L}_{0}=\mathcal{L}=\mathcal{L}_{1}$, then, our results reduce to the corresponding ones in Lemma 2.3 [6]. Otherwise, i.e., if strict inequality holds in (2.14), then our estimates (2.4), (2.5), (2.6) and (2.10) are more precise than the corresponding ones obtained from the preceding ones for $\mathcal{L}_{0}=\mathcal{L}$, or $\mathcal{L}_{0}=$ $\mathcal{L}_{1}$ ( see (3.1)-(3.4) given in [6]). This improvement is obtained under the same computational cost, since the computation of function $\mathcal{L}_{1}$ involves the computation of $\mathcal{L}_{0}$ or $\mathcal{L}$ (as a special case). Moreover, this modification leads to the advantages as already stated in the abstract of the paper.

Next, we complete this section with two auxiliary results involving functions appearing in the convergence analysis that follows in the next section.

Assumption 2.5. [8] Let

$$
\varphi(t):=\frac{1}{t^{\alpha}} \int_{0}^{t} \mathcal{L}(u) u^{\alpha-1} d u, \alpha \geq 1,0 \leq t \leq r
$$

where $\mathcal{L}$ is a positive integrable function and monotonically increasing in $[0, r]$. Then, function $\varphi$ is nondecreasing for each $\alpha$.

Assumption 2.6. [8] Let

$$
\psi(t):=\frac{1}{t^{2}} \int_{0}^{t} \mathcal{L}(u)(\alpha t-u) d u, \alpha \geq 1,0 \leq t \leq r,
$$

where $\mathcal{L}$ is as in Lemma 2.5. Then, function $\psi$ is monotonically increasing.

\section{Local convergence analysis}

We present the local convergence analysis of IGNM and IGNLM. The proof are analogous to the corresponding ones in [6], where we replace old estimates

$$
\begin{gathered}
\beta \int_{0}^{s(x)} \mathcal{L}(u) d u<1 \\
\int_{0}^{1}\left\|F^{\prime}\left(x^{\theta}\right)-F^{\prime}\left(x^{\star}\right)\right\| s(x) d \theta \leq \int_{0}^{s(x)} \mathcal{L}(u)(s(x)-u) d u \\
\left\|F^{\prime}(y)^{\dagger} F(x)\right\| \leq \frac{\left\|x-x^{\star}\right\|+\beta \int_{0}^{s(x)} \mathcal{L}(u)(s(x)-u) d u}{1-\beta \int_{0}^{s(y)} \mathcal{L}(u) d u}
\end{gathered}
$$


and

$$
\left\|F(x)^{\dagger} F(x)\right\| \leq s\left(x_{0}\right)+\frac{\beta \int_{0}^{s(x)} \mathcal{L}(u) d u}{1-\beta \int_{0}^{s(x)} \mathcal{L}(u) d u}
$$

by the new and more precise (2.4), (2.5), (2.6) and (2.10), respectively.

Next, we present the local convergence analysis of IGNM followed by the corresponding local convergence analysis of IGNLM.

THEOREM 3.1. Suppose $x^{\star}$ satisfies (1.1), $F$ has a continuous derivative, has full rank and $F^{\prime}(x)$ satisfies the radius Lipschitz condition with $\mathcal{L}$ average and the center-Lipschitz condition with $\mathcal{L}_{0}$ average where $\mathcal{L}$ and $\mathcal{L}_{0}$ are nondecreasing and (2.9) holds. Assume $B_{k}=F^{\prime}\left(x_{k}\right)^{T} F^{\prime}\left(x_{k}\right)$, for each $k$ in $(1.3)$

$v_{k}=\theta_{k}\left\|\left(P_{k} F^{\prime}\left(x_{k}\right)^{T} F^{\prime}\left(x_{k}\right)\right)^{-1}\right\|\left\|P_{k} F^{\prime}\left(x_{k}\right)^{T} F^{\prime}\left(x_{k}\right)\right\|=\theta_{k} \operatorname{Cond}\left(P_{k} F^{\prime}\left(x_{k}\right)^{T} F^{\prime}\left(x_{k}\right)\right)$

with $v_{k} \leq v<1$. Let $r_{0}>0$ satisfy

$$
(1+v) \frac{\beta \int_{0}^{r_{0}} \mathcal{L}(u) u d u}{r\left(1-\beta \int_{0}^{r_{0}} \mathcal{L}_{0}(u) d u\right)}+\frac{\sqrt{2} c \beta^{2} \int_{0}^{r_{0}} \mathcal{L}_{0}(u) d u}{r\left(1-\beta \int_{0}^{r_{0}} \mathcal{L}_{0}(u) d u\right)}+v \leq 1
$$

Then IGNM is convergent for all $x_{0} \in U\left(x^{\star}, r_{0}\right)$ and

$$
\begin{aligned}
\left\|x_{k+1}-x^{\star}\right\| \leq & (1+v) \frac{\beta \int_{0}^{s\left(x_{0}\right)} \mathcal{L}(u) d u}{s\left(x_{0}\right)^{2}\left(1-\beta \int_{0}^{s\left(x_{0}\right)} \mathcal{L}_{0}(u) d u\right)}\left\|x_{k}-x^{\star}\right\|^{2} \\
& +\left(\frac{\sqrt{2} c \beta^{2} \int_{0}^{s\left(x_{0}\right)} \mathcal{L}_{0}(u) d u}{s\left(x_{0}\right)\left(1-\beta \int_{0}^{s\left(x_{0}\right)} \mathcal{L}_{0}(u) d u\right)}+v\right)\left\|x_{k}-x^{\star}\right\|
\end{aligned}
$$

where $c=\left\|F\left(x^{\star}\right)\right\|, \beta=\left\|\left(F^{\prime}\left(x^{\star}\right)^{T} F^{\prime}\left(x^{\star}\right)\right)^{-1} F^{\prime}\left(x^{\star}\right)^{T}\right\|$,

$q=(1+v) \frac{\beta \int_{0}^{s\left(x_{0}\right)} \mathcal{L}(u) d u}{s\left(x_{0}\right)\left(1-\beta \int_{0}^{s\left(x_{0}\right)} \mathcal{L}_{0}(u) d u\right)}+\frac{\sqrt{2} c \beta^{2} \int_{0}^{s\left(x_{0}\right)} \mathcal{L}_{0}(u) d u}{s\left(x_{0}\right)\left(1-\beta \int_{0}^{s\left(x_{0}\right)} \mathcal{L}_{0}(u) d u\right)}+v<1$.

Proof. Let $x_{0} \in U\left(x^{\star}, r_{0}\right)$ where $r$ satisfies (2.8), from the monotonicity of $\mathcal{L}_{0}, \mathcal{L},(2.4)$ and Lemma 2.5, we have in turn

$$
\begin{aligned}
q & =(1+v) \frac{\beta \int_{0}^{s\left(x_{0}\right)} \mathcal{L}(u) d u}{s\left(x_{0}\right)^{2}\left(1-\beta \int_{0}^{s\left(x_{0}\right)} \mathcal{L}_{0}(u) d u\right)} s\left(x_{0}\right)+\frac{\sqrt{2} c \beta^{2} \int_{0}^{s\left(x_{0}\right)} \mathcal{L}_{0}(u) d u}{s\left(x_{0}\right)\left(1-\beta \int_{0}^{s\left(x_{0}\right)} \mathcal{L}_{0}(u) d u\right)}+v \\
& <(1+v) \frac{\sqrt{2} c \beta^{2} \int_{0}^{r_{0}} \mathcal{L}_{0}(u) d u}{s\left(x_{0}\right)\left(1-\beta \int_{0}^{r_{0}} \mathcal{L}(u) d u\right.}+v \leq 1
\end{aligned}
$$


and

$$
\begin{aligned}
\left\|\left[F^{\prime}\left(x^{\star}\right)^{T} F^{\prime}\left(x^{\star}\right)\right]^{-1} F^{\prime}\left(x^{\star}\right)^{T}\right\|\left\|F^{\prime}(x)-F^{\prime}\left(x^{\star}\right)\right\| & \leq \beta \int_{0}^{s\left(x_{0}\right)} \mathcal{L}_{0}(u) d u \\
& \leq \beta \int_{0}^{r_{0}} \mathcal{L}_{0}(u) d u<1, \\
& \quad \text { for each } x \in U\left(x^{\star}, r_{0}\right) .
\end{aligned}
$$

That is , $q$ given by (3.7) is less than 1 .

By Lemma 2.1 and 2.2, $F^{\prime}(x)$ has full rank for each $x \in U\left(x^{\star}, r_{0}\right)$ and

$$
\begin{aligned}
& \left\|\left[F^{\prime}(x)^{T} F^{\prime}(x)\right]^{-1} F^{\prime}(x)^{T}\right\| \leq \frac{\beta}{1-\beta \int_{0}^{s\left(x_{0}\right)} \mathcal{L}_{0}(u) d u}, \\
& \quad \text { for each } x \in U\left(x^{\star}, r_{0}\right)
\end{aligned}
$$

$$
\begin{aligned}
\left\|\left[F^{\prime}(x)^{T} F^{\prime}(x)\right]^{-1} F^{\prime}(x)^{T}-\left[F^{\prime}\left(x^{\star}\right)^{T} F^{\prime}\left(x^{\star}\right)\right]^{-1} F^{\prime}\left(x^{\star}\right)^{T}\right\| \leq & \frac{\sqrt{2} \beta^{2} \int_{0}^{s(x)} \mathcal{L}_{0}(u) d u}{1-\beta \int_{0}^{s(x)} \mathcal{L}_{0}(u) d u}, \\
& \text { for each } x \in U\left(x^{\star}, r_{0}\right) .
\end{aligned}
$$

Then, if $x_{k} \in U\left(x^{\star}, r_{0}\right)$, we have by (1.2) in turn that

$$
\begin{aligned}
x_{k+1}-x^{\star}= & x_{k}-x^{\star}-F^{\prime}\left(x_{k}\right)^{\dagger}\left(F\left(x_{k}\right)-F\left(x^{\star}\right)\right)+\left(F^{\prime}\left(x_{k}\right)^{T} F^{\prime}\left(x_{k}\right)\right)^{-1} r_{k} \\
= & F^{\prime}\left(x_{k}\right)^{\dagger} \int_{0}^{1}\left(F^{\prime}\left(x_{k}\right)-F^{\prime}\left(x^{\theta}\right)\right)\left(x_{k}-x^{\star}\right) d \theta \\
& +\left(F^{\prime}\left(x_{k}\right)^{T} F^{\prime}\left(x_{k}\right)\right)^{-1} P_{k}^{-1} P_{k} r_{k}+\left(F^{\prime}\left(x^{\star}\right)^{T} F^{\prime}\left(x^{\star}\right)\right)^{-1} F^{\prime}\left(x^{\star}\right)^{T} F\left(x^{\star}\right) \\
& -\left(F^{\prime}\left(x_{k}\right)^{T} F^{\prime}\left(x_{k}\right)\right)^{-1} F^{\prime}\left(x_{k}\right)^{T} F\left(x^{\star}\right) .
\end{aligned}
$$


Vol. LIV (2016) Extended local analysis of inexact Gauss-Newton ...

Hence, by Lemma 2.3 and conditions (2.8) and (2.3) we obtain

$$
\begin{aligned}
& \left\|x_{k+1}-x^{\star}\right\| \leq\left\|F^{\prime}\left(x_{k}\right)^{\dagger}\right\| \int_{0}^{1}\left\|F^{\prime}\left(x_{k}\right)-F^{\prime}\left(x^{\theta}\right)\right\|\left\|x_{k}-x^{\star}\right\| d \theta \\
& +\theta_{k}\left\|\left(F^{\prime}\left(x_{k}\right)^{T} F^{\prime}\left(x_{k}\right)\right)^{-1}\right\|\left\|P_{k} F^{\prime}\left(x_{k}\right)^{T} F^{\prime}\left(x_{k}\right)\right\| \\
& +\left\|\left(F^{\prime}\left(x_{k}\right)^{T} F^{\prime}\left(x_{k}\right)\right)^{-1} F^{\prime}\left(x^{\star}\right)^{T}-\left(F^{\prime}\left(x_{k}\right)^{T} F^{\prime}\left(x_{k}\right)\right)^{-1} F^{\prime}\left(x_{k}\right)^{T}\right\|\left\|F\left(x^{\star}\right)\right\| \\
& \leq \frac{\beta}{1-\beta \int_{0}^{s\left(x_{k}\right)} \mathcal{L}_{0}(u) d u} \\
& \times \int_{0}^{1} \int_{\theta s\left(x_{k}\right)}^{s\left(x_{k}\right)} \mathcal{L}(u) d u s\left(x_{k}\right) d \theta+\theta_{k}\left\|\left(P_{k} F^{\prime}\left(x_{k}\right)^{T} F^{\prime}\left(x_{k}\right)\right)^{-1}\right\| \\
& \times\left\|P_{k} F^{\prime}\left(x_{k}\right)^{T} F^{\prime}\left(x_{k}\right)\left(F^{\prime}\left(x_{k}\right)^{T} F^{\prime}\left(x_{k}\right)\right)^{-1} F^{\prime}\left(x_{k}\right)^{T} F\left(x_{k}\right)\right\| \\
& +\frac{\sqrt{2} c \beta^{2} \int_{0}^{s\left(x_{k}\right)} \mathcal{L}_{0}(u) d u}{1-\beta \int_{0}^{s\left(x_{k}\right)} \mathcal{L}_{0}(u) d u} \\
& \leq \frac{\beta \int_{0}^{s\left(x_{k}\right)} \mathcal{L}(u) d u}{1-\beta \int_{0}^{s\left(x_{k}\right)} \mathcal{L}_{0}(u) d u}+\theta_{k} \operatorname{Cond}\left(P_{k} F^{\prime}\left(x_{k}\right)^{T} F^{\prime}\left(x_{k}\right)\right) \\
& \times\left(s\left(x_{k}\right)+\frac{\beta \int_{0}^{s\left(x_{k}\right)} \mathcal{L}_{0}(u) d u}{1-\beta \int_{0}^{s\left(x_{k}\right)} \mathcal{L}_{0}(u) d u}\right) \\
& +\frac{\sqrt{2} c \beta^{2} \int_{0}^{s\left(x_{k}\right)} \mathcal{L}_{0}(u) d u}{1-\beta \int_{0}^{s\left(x_{k}\right)} \mathcal{L}_{0}(u) d u} \\
& \leq\left(1+v_{k}\right) \frac{\beta \int_{0}^{s\left(x_{k}\right)} \mathcal{L}(u) d u}{1-\beta \int_{0}^{s\left(x_{k}\right)} \mathcal{L}_{0}(u) d u}+v_{k} s\left(x_{k}\right) \\
& +\frac{\sqrt{2} c \beta^{2} \int_{0}^{s\left(x_{k}\right)} \mathcal{L}_{0}(u) d u}{1-\beta \int_{0}^{s\left(x_{k}\right)} \mathcal{L}_{0}(u) d u} .
\end{aligned}
$$

Taking $k=0$ above, we obtain $\left\|x_{1}-x^{\star}\right\| \leq q\left\|x_{0}-x^{\star}\right\|<\left\|x_{0}-x^{\star}\right\|$. That is $x_{1} \in U\left(x^{\star}, r_{0}\right)$. By mathematical induction, all $x_{k}$ belong to $U\left(x^{\star}, r_{0}\right)$ and 
$s\left(x_{k}\right)=\left\|x_{k}-x^{\star}\right\|$ decreases monotonically. Therefore, for all $k \geq 0$, we have

$$
\begin{aligned}
\left\|x_{k+1}-x^{\star}\right\| \leq & \left(1+v_{k}\right) \frac{\beta \int_{0}^{s\left(x_{k}\right)} \mathcal{L}(u) d u}{s\left(x_{k}\right)^{2}\left(1-\beta \int_{0}^{s\left(x_{k}\right)} \mathcal{L}_{0}(u) d u\right)} s\left(x_{k}\right)^{2} \\
& +v_{k} s\left(x_{k}\right)+\frac{\sqrt{2} c \beta^{2} \int_{0}^{s\left(x_{k}\right)} \mathcal{L}_{0}(u) d u}{1-\beta \int_{0}^{s\left(x_{k}\right)} \mathcal{L}_{0}(u) d u} \\
\leq & (1+v) \frac{\beta \int_{0}^{s\left(x_{k}\right)} \mathcal{L}(u) d u}{s\left(x_{k}\right)^{2}\left(1-\beta \int_{0}^{s\left(x_{k}\right)} \mathcal{L}_{0}(u) d u\right)} s\left(x_{k}\right)^{2} \\
& +\left(v+\frac{\sqrt{2} c \beta^{2} \int_{0}^{s\left(x_{k}\right)} \mathcal{L}_{0}(u) d u}{1-\beta \int_{0}^{s\left(x_{k}\right)} \mathcal{L}_{0}(u) d u}\right) s\left(x_{k}\right) .
\end{aligned}
$$

THEOREM 3.2. Suppose $x^{\star}$ satisfies (1.1), $F\left(x^{\star}\right)=0, F$ has a continuous derivative, has full rank and $F^{\prime}(x)$ satisfies the center Lipschitz condition (2.3) with $\mathcal{L}_{0}$ average where $\mathcal{L}_{0}$ is nondecreasing and (2.9) holds. Assume $B_{k}=F^{\prime}\left(x_{0}\right)^{T} F^{\prime}\left(x_{0}\right)$, for each $k$ in (1.3),

$v_{k}=\theta_{k}\left\|\left(P_{0} F^{\prime}\left(x_{0}\right)^{T} F^{\prime}\left(x_{0}\right)\right)^{-1}\right\|\left\|P_{0} F^{\prime}\left(x_{0}\right)^{T} F^{\prime}\left(x_{0}\right)\right\|=\theta_{k} \operatorname{Cond}\left(P_{0} F^{\prime}\left(x_{0}\right)^{T} F^{\prime}\left(x_{0}\right)\right)$

with $v_{k} \leq v<1$. Let $r_{0}>0$ satisfy

$$
(1+v) \frac{\beta \int_{0}^{r_{0}} \mathcal{L}_{0}(u)\left(r_{0}-u\right) d u}{r\left(1-\beta \int_{0}^{r_{0}} \mathcal{L}_{0}(u) d u\right)}+\frac{v+\beta \int_{0}^{r_{0}} \mathcal{L}_{0}(u) d u}{\left.1-\beta \int_{0}^{r_{0}} \mathcal{L}_{0}(u) d u\right)} \leq 1 .
$$

Then Modified Inexact Gauss-Newton Method(MIGNM) is convergent for all $x_{0} \in U\left(x^{\star}, r_{0}\right)$ and

$$
\begin{aligned}
\left\|x_{k+1}-x^{\star}\right\| \leq & (1+v) \frac{\beta \int_{0}^{s\left(x_{0}\right)} \mathcal{L}_{0}(u)\left(s\left(x_{0}\right)-u\right) d u}{s\left(x_{0}\right)^{2}\left(1-\beta \int_{0}^{s\left(x_{0}\right)} \mathcal{L}_{0}(u) d u\right)}\left\|x_{k}-x^{\star}\right\|^{2} \\
& +\frac{v+\beta \int_{0}^{s\left(x_{0}\right)} \mathcal{L}_{0}(u) d u}{1-\beta \int_{0}^{s\left(x_{0}\right)} \mathcal{L}_{0}(u) d u}
\end{aligned}
$$

is less than 1 .

Proof. Simply replace $\mathcal{L}_{1}$ by $\mathcal{L}_{0}$ in the proof of Theorem 3.2 in [6] or see the proof in [1].

Next, we preset the corresponding results for Inexact Gauss-Newton-Like Method IGNLM, where $B_{k}=B\left(x_{k}\right)$ approximates $F^{\prime}\left(x_{k}\right)^{T} F^{\prime}\left(x_{k}\right)$. 
THEOREM 3.3. Suppose $x^{\star}$ satisfies (1.1), $F$ has a continuous derivative, full rank and $F^{\prime}(x)$ satisfies the radius Lipschitz condition (2.8) with $\mathcal{L}$ average and the center Lipschitz condition with $\mathcal{L}_{0}$ average where $\mathcal{L}$ and $\mathcal{L}_{0}$ are nondecreasing and $(2.9)$ holds. Let $B(x)$ be invertible and

$$
\begin{aligned}
& \qquad\left\|B(x)^{-1} F^{\prime}(x)^{T} F^{\prime}(x)\right\| \leq \omega_{1}, \quad\left\|B(x)^{-1} F^{\prime}(x)^{T} F^{\prime}(x)-I\right\| \leq \omega_{2}, \\
& v_{k}=\theta_{k}\left\|\left(P_{k} F^{\prime}\left(x_{k}\right)^{T} F^{\prime}\left(x_{k}\right)\right)^{-1}\right\|\left\|P_{k} F^{\prime}\left(x_{k}\right)^{T} F^{\prime}\left(x_{k}\right)\right\|=\theta_{k} \operatorname{Cond}\left(P_{k} F^{\prime}\left(x_{k}\right)^{T} F^{\prime}\left(x_{k}\right)\right) \\
& \text { with } v_{k} \leq v<1 \text {. Let } r_{0}>0 \text { satisfy }
\end{aligned}
$$

$$
(1+v) \frac{\beta \omega_{1} \int_{0}^{r_{0}} \mathcal{L}(u) u d u}{r_{0}\left(1-\beta \int_{0}^{r_{0}} \mathcal{L}_{0}(u) d u\right)}+\omega_{2}+\omega_{1} v+\frac{\sqrt{2} \beta^{2} \omega_{1} \int_{0}^{r_{0}} \mathcal{L}_{0}(u) d u}{\left.r_{0}\left(1-\beta \int_{0}^{r_{0}} \mathcal{L}_{0}(u) d u\right)\right)} \leq 1
$$

Then IGNLM is convergent for all $x_{0} \in U\left(x^{\star}, r_{0}\right)$ and

$$
\begin{aligned}
\left\|x_{k+1}-x^{\star}\right\| \leq & (1+v) \frac{\beta \omega_{1} \int_{0}^{s\left(x_{0}\right)} \mathcal{L}(u) u d u}{s\left(x_{0}\right)^{2}\left(1-\beta \int_{0}^{s\left(x_{0}\right)} \mathcal{L}_{0}(u) d u\right)}\left\|x_{k}-x^{\star}\right\|^{2} \\
& +\left(\omega_{2}+\omega_{1} v+\frac{\sqrt{2} \beta^{2} \omega_{1} \int_{0}^{s\left(x_{0}\right)} \mathcal{L}_{0}(u) d u}{s\left(x_{0}\right)\left(1-\beta \int_{0}^{s\left(x_{0}\right)} \mathcal{L}_{0}(u) d u\right)}\right)\left\|x_{k}-x^{\star}\right\|
\end{aligned}
$$

where $c=\left\|F\left(x^{\star}\right)\right\|, \beta=\left\|\left[F^{\prime}\left(x^{\star}\right)^{T} F^{\prime}\left(x^{\star}\right)\right]^{-1} F^{\prime}\left(x^{\star}\right)^{T}\right\|$,

$$
\begin{aligned}
q= & (1+v) \frac{\beta \omega_{1} \int_{0}^{s\left(x_{0}\right)} \mathcal{L}(u) u d u}{s\left(x_{0}\right)\left(1-\beta \int_{0}^{s\left(x_{0}\right)} \mathcal{L}_{0}(u) d u\right)} \\
& +\omega_{2}+\omega_{1} v+\frac{\sqrt{2} \beta^{2} \omega_{1} \int_{0}^{s\left(x_{0}\right)} \mathcal{L}_{0}(u) d u}{s\left(x_{0}\right)\left(1-\beta \int_{0}^{s\left(x_{0}\right)} \mathcal{L}_{0}(u) d u\right)}
\end{aligned}
$$

is less than 1.

Proof. Let $x_{0} \in U\left(x^{\star}, r_{0}\right)$, where $r_{0}$ satisfies (3.11), by the monotonicity of $\mathcal{L}_{0}, \mathcal{L},(2.14)$ and Lemma 2.5), we have

$$
\begin{aligned}
q= & (1+v) \frac{\beta \omega_{1} \int_{0}^{s\left(x_{0}\right)} \mathcal{L}(u) u d u}{s\left(x_{0}\right)^{2}\left(1-\beta \int_{0}^{s\left(x_{0}\right)} \mathcal{L}_{0}(u) d u\right)} s\left(x_{0}\right) \\
& +\omega_{2}+\omega_{1} v+\frac{\sqrt{2} \beta^{2} \omega_{1} \int_{0}^{s\left(x_{0}\right)} \mathcal{L}_{0}(u) d u}{s\left(x_{0}\right)\left(1-\beta \int_{0}^{s\left(x_{0}\right)} \mathcal{L}_{0}(u) d u\right)} \\
< & (1+v) \frac{\beta \omega_{1} \int_{0}^{r_{0}} \mathcal{L}(u) u d u}{r_{0}^{2}\left(1-\beta \int_{0}^{r_{0}} \mathcal{L}_{0}(u) d u\right)} r_{0} \\
& +\omega_{2}+\omega_{1} v+\frac{\sqrt{2} \beta^{2} \omega_{1} \int_{0}^{r_{0}} \mathcal{L}_{0}(u) d u}{r_{0}\left(1-\beta \int_{0}^{r_{0}} \mathcal{L}_{0}(u) d u\right)} \leq 1,
\end{aligned}
$$


and

$$
\begin{aligned}
\left\|\left[F^{\prime}(x)^{T} F^{\prime}(x)\right]^{-1} F^{\prime}(x)^{T}\right\|\left\|F^{\prime}(x)-F^{\prime}\left(x^{\star}\right)\right\| & \leq \beta \int_{0}^{s(x)} \mathcal{L}_{0}(u) d u \\
& \leq \beta \int_{0}^{r_{0}} \mathcal{L}_{0}(u) d u<1, \\
& \text { for each } x \in U\left(x^{\star}, r_{0}\right) .
\end{aligned}
$$

That is, $q$ given by (3.13) is less than 1 .

Since $F^{\prime}(x), x \in U\left(x^{\star}, r_{0}\right)$ has full rank by Lemma 2.1 and 2.2 , we have

$$
\begin{aligned}
& \left\|\left[F^{\prime}(x)^{T} F^{\prime}(x)\right]^{-1} F^{\prime}(x)^{T}\right\| \leq \frac{\beta}{1-\beta \int_{0}^{s(x)} \mathcal{L}_{0}(u) d u}, \text { for each } x \in U\left(x^{\star}, r_{0}\right) \\
& \left\|\left[F^{\prime}(x)^{T} F^{\prime}(x)\right]^{-1} F^{\prime}(x)^{T}-\left[F^{\prime}\left(x^{\star}\right)^{T} F^{\prime}\left(x^{\star}\right)\right]^{-1} F^{\prime}\left(x^{\star}\right)^{T}\right\| \leq \\
& \begin{aligned}
& \frac{\sqrt{2} \beta^{2} \int_{0}^{s(x)} \mathcal{L}_{0}(u) d u}{1-\beta \int_{0}^{s(x)} \mathcal{L}_{0}(u) d u}, \\
& \text { for each } x \in U\left(x^{\star}, r_{0}\right) .
\end{aligned}
\end{aligned}
$$

Then, if $x_{k} \in U\left(x^{\star}, r_{0}\right)$, we have by (1.2) in turn that

$$
\begin{aligned}
x_{k+1}-x^{\star}= & x_{k}-x^{\star}-B_{k}^{-1} F^{\prime}\left(x_{k}\right)^{T}\left(F\left(x_{k}\right)-F\left(x^{\star}\right)\right)+B_{k}^{-1} r_{k} \\
= & x_{k}-x^{\star}-\int_{0}^{1} B_{k}^{-1} F^{\prime}\left(x_{k}\right)^{T} F^{\prime}\left(x^{\theta}\right)\left(x_{k}-x^{\star}\right) d \theta \\
& +B_{k}^{-1} P_{k}^{-1} P_{k} r_{k}+B_{k}^{-1} F^{\prime}\left(x_{k}\right)^{T} F\left(x_{k}\right)\left\{\left[F^{\prime}\left(x^{\star}\right)^{T} F^{\prime}\left(x^{\star}\right)\right]^{-1} F^{\prime}\left(x^{\star}\right)^{T} F\left(x^{\star}\right)\right. \\
& \left.-\left(F^{\prime}\left(x_{k}\right)^{T} F^{\prime}\left(x_{k}\right)\right)^{-1} F^{\prime}\left(x_{k}\right)^{T} F\left(x^{\star}\right)\right\} \\
= & B_{k}^{-1} F^{\prime}\left(x_{k}\right)^{T} F^{\prime}\left(x_{k}\right) \int_{0}^{1} F^{\prime}\left(x_{k}\right)^{\dagger}\left(F^{\prime}\left(x_{k}\right)-F^{\prime}\left(x^{\theta}\right)\right)\left(x_{k}-x^{\star}\right) d \theta \\
& -B_{k}^{-1}\left(F^{\prime}\left(x_{k}\right)^{T} F^{\prime}\left(x_{k}\right)-B_{k}\right)\left(x_{k}-x^{\star}\right)+B_{k}^{-1} P_{k}^{-1} P_{k} r_{k} \\
& +B_{k}^{-1} F^{\prime}\left(x_{k}\right)^{T} F\left(x_{k}\right)\left\{\left[F^{\prime}\left(x^{\star}\right)^{T} F^{\prime}\left(x^{\star}\right)\right]^{-1} F^{\prime}\left(x^{\star}\right)^{T} F\left(x^{\star}\right)\right. \\
& \left.-\left(F^{\prime}\left(x_{k}\right)^{T} F^{\prime}\left(x_{k}\right)\right)^{-1} F^{\prime}\left(x_{k}\right)^{T} F\left(x^{\star}\right)\right\} .
\end{aligned}
$$


Vol. LIV (2016) Extended local analysis of inexact Gauss-Newton ...

In vieq of Lemma 2.3 and conditions (2.8) and (2.3) we obtain

$$
\begin{aligned}
& \left\|x_{k+1}-x^{\star}\right\| \leq\left\|B_{k}^{-1} F^{\prime}\left(x_{k}\right)^{T} F^{\prime}\left(x_{k}\right)\right\|\left\|\int_{0}^{1}\right\| F^{\prime}\left(x_{k}\right)^{\dagger}\|\| F^{\prime}\left(x_{k}\right)-F^{\prime}\left(x^{\theta}\right)\|\| x_{k}-x^{\star} \| d \theta \\
& +\left\|B_{k}^{-1}\left(F^{\prime}\left(x_{k}\right)^{T} F^{\prime}\left(x_{k}\right)-B_{k}\right)\right\|\left\|x_{k}-x^{\star}\right\| \\
& +\theta_{k}\left\|B_{k}^{-1} P_{k}^{-1}\right\|\left\|P_{k} F^{\prime}\left(x_{k}\right)^{T} F^{\prime}\left(x_{k}\right)\right\| \\
& +\left\|B_{k}^{-1} F^{\prime}\left(x_{k}\right)^{T} F^{\prime}\left(x_{k}\right)\right\| \|\left[F^{\prime}\left(x^{\star}\right)^{T} F^{\prime}\left(x^{\star}\right)\right]^{-1} F^{\prime}\left(x^{\star}\right)^{T} \\
& -\left[F^{\prime}\left(x_{k}\right)^{T} F^{\prime}\left(x_{k}\right)\right]^{-1} F^{\prime}\left(x_{k}\right)^{T}\|\| F^{\prime}\left(x^{\star}\right) \| \\
& \leq \frac{\beta \omega_{1}}{1-\beta \int_{0}^{s\left(x_{k}\right)} \mathcal{L}_{0}(u) d u} \int_{0}^{1} \int_{\theta s\left(x_{k}\right)}^{s\left(x_{k}\right)} \mathcal{L}(u) d u s\left(x_{k}\right) d \theta \\
& +\omega_{2} s\left(x_{k}\right)+\theta_{k}\left\|B_{k}^{-1} F^{\prime}\left(x_{k}\right)^{T} F^{\prime}\left(x_{k}\right)\right\| \\
& \times\left\|\left(P_{k} F^{\prime}\left(x_{k}\right)^{T} F^{\prime}\left(x_{k}\right)\right)^{-1}\right\|\left\|F^{\prime}\left(x_{k}\right)^{\dagger} F\left(x_{k}\right)\right\| \\
& +\frac{\sqrt{2} c \beta^{2} \omega_{1} \int_{0}^{s\left(x_{k}\right)} \mathcal{L}_{0}(u) d u}{1-\beta \int_{0}^{s\left(x_{k}\right)} \mathcal{L}_{0}(u) d u} \\
& \leq \frac{\beta \omega_{1} \int_{0}^{s\left(x_{k}\right)} \mathcal{L}(u) d u}{1-\beta \int_{0}^{s\left(x_{k}\right)} \mathcal{L}_{0}(u) d u} \\
& +\omega_{1} v_{k}\left(s\left(x_{k}\right)+\frac{\beta \int_{0}^{s\left(x_{k}\right)} \mathcal{L}_{0}(u) d u}{1-\beta \int_{0}^{s\left(x_{k}\right)} \mathcal{L}_{0}(u) d u}\right) \\
& +\omega_{2} s\left(x_{k}\right)+\frac{\sqrt{2} c \beta^{2} \omega_{1} \int_{0}^{s\left(x_{k}\right)} \mathcal{L}_{0}(u) d u}{1-\beta \int_{0}^{s\left(x_{k}\right)} \mathcal{L}_{0}(u) d u} \\
& \leq\left(1+v_{k}\right) \frac{\beta \omega_{1} \int_{0}^{s\left(x_{k}\right)} \mathcal{L}(u) d u}{1-\beta \int_{0}^{s\left(x_{k}\right)} \mathcal{L}_{0}(u) d u}+\left(\omega_{2}+\omega_{1} v_{k}\right) s\left(x_{k}\right) \\
& +\frac{\sqrt{2} c \beta^{2} \omega_{1} \int_{0}^{s\left(x_{k}\right)} \mathcal{L}_{0}(u) d u}{1-\beta \int_{0}^{s\left(x_{k}\right)} \mathcal{L}_{0}(u) d u} .
\end{aligned}
$$

If $k=0$ above, we obtain $\left\|x_{1}-x^{\star}\right\| \leq q\left\|x_{0}-x^{\star}\right\|<\left\|x_{0}-x^{\star}\right\|$. That is $x_{1} \in$ $U\left(x^{\star}, r_{0}\right)$, this shows that (1.2) can be continued an infinite number of times. By mathematical induction, all $x_{k}$ belong to $U\left(x^{\star}, r_{0}\right)$ and $s\left(x_{k}\right)=\left\|x_{k}-x^{\star}\right\|$ 
decreases monotonically. Therefore, for all $k \geq 0$, we have

$$
\begin{aligned}
\left\|x_{k+1}-x^{\star}\right\| \leq & \left(1+v_{k}\right) \frac{\beta \omega_{1} \int_{0}^{s\left(x_{k}\right)} \mathcal{L}(u) d u}{s\left(x_{k}\right)^{2}\left(1-\beta \int_{0}^{s\left(x_{k}\right)} \mathcal{L}_{0}(u) d u\right)} s\left(x_{k}\right)^{2} \\
& +\left(\omega_{2}+\omega_{1} v_{k}\right) s\left(x_{k}\right)+\frac{\sqrt{2} c \beta^{2} \omega_{1} \int_{0}^{s\left(x_{k}\right)} \mathcal{L}_{0}(u) d u}{s\left(x_{k}\right)\left(1-\beta \int_{0}^{s\left(x_{k}\right)} \mathcal{L}_{0}(u) d u\right)} s\left(x_{k}\right) \\
\leq & (1+v) \frac{\beta \omega_{1} \int_{0}^{s\left(x_{0}\right)} \mathcal{L}(u) d u}{s\left(x_{0}\right)^{2}\left(1-\beta \int_{0}^{s\left(x_{0}\right)} \mathcal{L}_{0}(u) d u\right)} s\left(x_{k}\right)^{2} \\
& +\left(\omega_{2}+\omega_{1} v+\frac{\sqrt{2} c \beta^{2} \omega_{1} \int_{0}^{s\left(x_{0}\right)} \mathcal{L}_{0}(u) d u}{s\left(x_{0}\right)\left(1-\beta \int_{0}^{s\left(x_{0}\right)} \mathcal{L}_{0}(u) d u\right)}\right) s\left(x_{k}\right) .
\end{aligned}
$$

REMARK 3.4. If $\mathcal{L}_{0}=\mathcal{L}_{1}$ our results reduce to the corresponding ones in [6] which in turn improved earlier ones $[7,8,11]$. Otherwise, i.e., if $\mathcal{L}_{0}<\mathcal{L}_{1}$ or $\mathcal{L}_{0}<\mathcal{L}$, our results constitute an improvement with advantages as already stated in the abstract of this paper.

\section{Applications and Examples}

Let all " $\mathcal{L}$ " functions be positive constant functions. Then, the following corollaries are obtained.

COROLLARY 4.1. Suppose $x^{\star}$ satisfies (1.1), $F$ has a continuous derivative, has full rank and $F^{\prime}(x)$ satisfies the radius Lipschitz condition with $\mathcal{L}$ average

$$
\left\|F^{\prime}(x)-F^{\prime}\left(x^{\theta}\right)\right\| \leq(1-\theta) \mathcal{L}\left\|x-x^{\star}\right\|, \quad 0 \leq \theta \leq 1,
$$

and the center Lipschitz condition with $\mathcal{L}_{0}$ average

$$
\left\|F^{\prime}(x)-F^{\prime}\left(x^{\star}\right)\right\| \leq \mathcal{L}_{0} \theta\left\|x-x^{\star}\right\|,
$$

where $x^{\theta}=x^{\star}+\theta\left(x-x^{\star}\right), s(x)=\left\|x-x^{\star}\right\|$ and (2.9) holds. Assume $B_{k}=$ $F^{\prime}\left(x_{k}\right)^{T} F^{\prime}\left(x_{k}\right)$ for each $k$ in $(1.2), v_{k}=\theta_{k}\left\|\left[P_{k} F^{\prime}\left(x_{k}\right)^{T} F^{\prime}\left(x_{k}\right)\right]^{-1}\right\|=\theta_{k}$ Cond $\left(P_{k} F^{\prime}\left(x_{k}\right)^{T} F^{\prime}\left(x_{k}\right)\right)$ with $v_{k} \leq v<1$. Let $r_{0}>0$ satisfy

$$
r_{0}=\frac{2\left(1-v-\sqrt{2} c \mathcal{L}_{0} \beta^{2}\right.}{\beta\left[(1+v) \mathcal{L}+2(1-v) \mathcal{L}_{0}\right]}
$$


where $c=\left\|F\left(x^{\star}\right)\right\|, \beta=\left\|\left[F^{\prime}\left(x^{\star}\right)^{T} F^{\prime}\left(x^{\star}\right)\right]^{-1} F^{\prime}\left(x^{\star}\right)^{T}\right\|$. Then (IGNM) is convergent for all $x_{0} \in U\left(x^{\star}, r_{0}\right)$,

$$
q=v+\frac{\mathcal{L} \beta(1+v)\left\|x_{0}-x^{\star}\right\|+2 \sqrt{2} c \mathcal{L}_{0} \beta^{2}}{2\left(1-\mathcal{L}_{0} \beta\left\|x_{0}-x^{\star}\right\|\right)}<1,
$$

and the inequality (3.6) holds.

COROLLARY 4.2. Suppose $x^{\star}$ satisfies $(1.1), F\left(x^{\star}\right)=0, F$ has a continuous derivative, full rank and $F^{\prime}(x)$ satisfies the center Lipschitz condition with Lipschitz condition with $\mathcal{L}_{0}$ average and (2.9) holds. Assume $B_{k}=F^{\prime}\left(x_{0}\right)^{T} F^{\prime}\left(x_{0}\right)$ for each $k$ in $(1.2)$,

$v_{k}=\theta_{k}\left\|\left[P_{0} F^{\prime}\left(x_{0}\right)^{T} F^{\prime}\left(x_{0}\right)\right]^{-1}\right\|\left\|P_{0} F^{\prime}\left(x_{0}\right)^{T} F^{\prime}\left(x_{0}\right)\right\|=\theta_{k} \operatorname{Cond}\left(P_{0} F^{\prime}\left(x_{0}\right)^{T} F^{\prime}\left(x_{0}\right)\right)$

with $v_{k} \leq v<1$. Let $r_{0}>0$ satisfy

$$
r_{0}=\frac{2(1-v)}{\beta \mathcal{L}_{0}(5+v)}
$$

where $\beta=\left\|\left[F^{\prime}\left(x^{\star}\right)^{T} F^{\prime}\left(x^{\star}\right)\right]^{-1} F^{\prime}\left(x^{\star}\right)^{T}\right\|$. Then (MIGNM) is convergent for all $x_{0} \in U\left(x^{\star}, r_{0}\right)$,

$$
q=\frac{\mathcal{L}_{0} \beta\left\|x_{0}-x^{\star}\right\|+v}{1-\mathcal{L}_{0} \beta\left\|x_{0}-x^{\star}\right\|}+\frac{\mathcal{L}_{0} \beta\left\|x_{0}-x^{\star}\right\|(1+v)}{2\left(1-\mathcal{L}_{0} \beta\left\|x_{0}-x^{\star}\right\|\right)}<1,
$$

and the inequality (3.4) holds.

COROLLARY 4.3. Suppose $x^{\star}$ satisfies (1.1), $F$ has a continuous derivative in $U\left(x^{\star}, r\right) F^{\prime}\left(x_{k}\right)$ has full rank and $F^{\prime}(x)$ satisfies the radius Lipschitz condition (4.1) with $\mathcal{L}$ average and the center Lipschitz condition (4.2) with $\mathcal{L}_{0}$ average and (2.9) holds. Assume $B(x)$ and $F^{\prime}(x)^{T} F^{\prime}(x)$ satisfy (3.10),

$v_{k}=\theta_{k}\left\|\left[P_{k} F^{\prime}\left(x_{k}\right)^{T} F^{\prime}\left(x_{k}\right)\right]^{-1}\right\|\left\|P_{k} F^{\prime}\left(x_{k}\right)^{T} F^{\prime}\left(x_{k}\right)\right\|=\theta_{k} \operatorname{Cond}\left(P_{k} F^{\prime}\left(x_{k}\right)^{T} F^{\prime}\left(x_{k}\right)\right)$

with $v_{k} \leq v<1$. Let $r_{0}>0$ satisfy

$$
r_{0}=\frac{2\left(1-v \omega_{1}-\omega_{2}-\sqrt{2} c \mathcal{L}_{0} \beta^{2} \omega_{1}\right.}{\beta\left[(1+v) \omega_{1} \mathcal{L}+2\left(1-\omega_{2}-\omega_{1} v\right) \mathcal{L}_{0}\right]}
$$

where $c=\left\|F\left(x^{\star}\right)\right\|, \beta=\left\|\left[F^{\prime}\left(x^{\star}\right)^{T} F^{\prime}\left(x^{\star}\right)\right]^{-1} F^{\prime}\left(x^{\star}\right)^{T}\right\|$. Then IGNLM is convergent for all $x_{0} \in U\left(x^{\star}, r_{0}\right)$,

$$
q=\omega_{1} v+\omega_{2}+\frac{\mathcal{L} \beta \omega_{1}(1+v)\left\|x_{0}-x^{\star}\right\|+2 \sqrt{2} c \mathcal{L}_{0} \beta^{2} \omega_{1}}{2\left(1-\mathcal{L}_{0} \beta\left\|x_{0}-x^{\star}\right\|\right)}<1,
$$

and the inequality (3.12) holds. 
REMARK 4.4. (a) If, $\mathcal{L}_{0}=\mathcal{L}_{1}$ the results reduce to the corresponding ones in [6].

(b) The results of section 5 in [6-8] using only center-Lipschitz condition can be improved with $\mathcal{L}_{0}$ replacing $\mathcal{L}$.

Next, we complete this study with a numerical example where $\mathcal{L}_{0}<\mathcal{L}$.

EXAMPLE 4.5. Let $m=n=3, x=y=\mathbb{R}^{3}, \mathcal{D}=\bar{U}(0,1)$ and $x^{\star}=$ $(0,0,0)^{T}$. Define function $F$ on $\mathcal{D}$ for $w=(x, y, z)^{T}$ by

$$
F(w)=\left(e^{x}-1, \frac{e-1}{2} y^{2}+y, z\right)^{T} .
$$

Then, the Fréchet derivative of $F$ is given by

$$
F^{\prime}(w)=\left(\begin{array}{ccc}
e^{x} & 0 & 0 \\
0 & (e-1) y+1 & 0 \\
0 & 0 & 1
\end{array}\right)
$$

Notice that we have $F\left(x^{\star}\right)=0, F^{\prime}\left(x^{\star}\right)=F^{\prime}\left(x^{\star}\right)^{-1}=\operatorname{diag}\{1,1,1\}, \beta=1$ and $\mathcal{L}_{0}=e-1<\mathcal{L}=e^{\frac{1}{\mathcal{L}_{0}}}<\mathcal{L}_{1}=e$, where $r_{0}=\frac{1}{\mathcal{L}_{0}}<r=1$. Estimate (2.9) holds as a strict inequality. Therefore, the new error bounds are more precise than the old ones using only $\mathcal{L}_{1}[7-9]$ or $\mathcal{L}_{0}$ and $\mathcal{L}_{1}[4,5]$.

More examples where $\mathcal{L}_{0}<\mathcal{L}_{1}$ or $\mathcal{L}_{0}<\mathcal{L}$ can be found in [2]- [5].

\section{Conclusions}

Under the hypothesis that $F^{\prime}\left(x^{\star}\right) F^{\prime}$ satisfies certain Lipschitz conditions, we presented a more precise local convergence analysis for the inexact GaussNewton method under the same computational cost as in earlier studies such as $[3,4,6-8,11]$ with advantages as stated in the abstract of this paper using our new idea of restricted convergence domains. Numerical examples are provided to show these advantages.

\section{References}

[1] I.K. Argyros, Computational theory of iterative methods, Studies in Computational Mathematics (15), C.K. Chui and L. Wuytack (Eds.), Elsevier Publ. Co., New York, U.S.A, 2007. 
[2] I.K. Argyros, Y.J.Cho, and S.Hilout, Numerical methods for equations and its applications, CRC Press, Taylor and Francis, New York, 2012.

[3] I.K. Argyros and S. Hilout, Weak convergence conditions for inexact Newton-type methods, Appl.Math. Comput. 218, (2011), 2800-2809.

[4] I.K. Argyros and S. Hilout, Extending the applicability of the Gauss-Newton method under average Lipschitz-type conditions, Numer. Algorithms, 58, (2011), 2352.

[5] I.K. Argyros and S. Hilout, Weaker conditions for the convergence of Newton's method, J. Complexity, 28, (2012), 364-387.

[6] J. Chen, The convergence analysis of inexact Gauss-Newton methods for nonlinear problems, Comput. Optim. Appl. 40, (2008), 97-118.

[7] J. Chen and W. Li, Convergence of Gauss-Newton's method and uniqueness of the solution, Appl. Math. Comput. 170, (2005), 686-705.

[8] J.Chen and W. Li, Convergence behaviour of inexact Newton methods under weaker Lipschitz condition, J. Comput. Appl. Math. 191, (2006), 143-164.

[9] R.S.Dembo, S.C. Eisenstat, and T. Steihaus, Inexact Newton methods, SIAM J. Numer. Anal. 19, (1982), 400-408.

[10] J.E.Jr. Dennis and R.B.Schnabel, Numerical methods for unconstrained optimization and nonlinear equations (Corrected reprint of the 1983 original), Classics in Appl. Math. (16), SIAM, Philadelphia, PA, 1996.

[11] B. Morini, Convergence behaviour of inexact Newton method, Math. Comp. 61, (1999), 1605-1613.

[12] J.M. Ortega and W.C. Rheinholdt, Iterative Solution of Nonlinear Equations in Several Variables, Academic Press, New York, 1970.

[13] G.W.Stewart, On the continuity of the generalized inverse, SIAM J. Appl. Math. 17, (1969), 33-45.

[14] W.C. Rheinholdt, An adaptive continuation process for solving systems of nonlinear equations, Polish Academy of Science, Banach Ctr. Publ. 3, (1977), 129-142.

[15] J.F. Traub, Iterative methods for the solution of equations, Prentice- Hall Series in Automatic Computation, Englewood Cliffs, N. J. 1964.

Ioannis K. Argyros

Department of Mathematical Sciences, Cameron University

Lawton, OK 73505, USA

E-mail: iargyros@cameron.edu

Santhosh George

Department ofMathematical and Computational Sciences, NIT Karnataka

India-575 025

E-mail: sgeorge@nitk.ac.in

Received: 16.02.2016

Accepted: 17.06.2016 